\title{
CHOLERETIC PROPERTIES OF URSODEOXYCHOLIC ACID AND CHENODEOXYCHOLIC ACID IN DOGS
}

\author{
Saizo YANAURA and Shigeru ISHIKAWA \\ Department of Pharmacology, Hoshi College of Pharmacy, \\ Ehara, Shinagawa-ku, Tokyo 142, Japan
}

Accepted December 21, 1977

\begin{abstract}
Choleretic effects and properties of ursodeoxycholic and chenodeoxycholic acids given orally were investigated in comparison with dehydrocholic acid in conscious Jogs with cholecystectomy. Ursodeoxycholic acid as well as chenodeoxycholic acid increased the bile flow and the concentrations of phospholipid, cholesterol and bile acids in the bile. After administration of either ursodeoxycholic acid or chenodeoxycholic acid, a great amount of each bilc acid appcared in the bile. Ursodeoxycholic and chenodeoxycholic acids increased the outputs of phospholipid, cholesterol and bilirubin in the bile. On the other hand, dehydrocholic acid markedly decreased the concentrations and outputs of all the above materials in the bile, despite a considerable increase in the bile flow. After administration of dehydrocholic acid, $3 \alpha, 7 \alpha$-dihydroxy12-keto-cholanoic acid appearcd in the bile instead of dehydrocholic acid. The results suggest that ursodeoxycholic and chenodcoxycholic acids are transported into the bile from the hepatic cells where they produce a choleresis due to the bile acid-dependent mechanism. Dehydrocholic acid is metabolized in the liver and the metabolites produce hydrocholeresis.
\end{abstract}

The important role of bile acids in stimulating bile flow is well known. An apparently linear relationship has been shown between bile acid secretion rate and bile flow (1). The biliary excretions of phospholipid and cholesterol also appear to be dependent upon bile acid secretion (2). Such a bile secretory mechanism associated with the bile acid secretion is termed 'bile acid-dependent fraction' in the bile formation (3). On the other hand, the existence of the fraction in the bile formation which is not dependent on the bile acid secretion has been revealed $(4,5)$.

Klaassen showed that there was not a good inverse correlation between the micellar properties of bile acids and the ability to increase bile flow and suggested that there is a difference among the choleretic properties of bile acid (6). There are, however, few reports on ursodeoxycholic and chenodeoxycholic acids, unlike cholic, deoxycholic and dehydrocholic acids and their conjugates, and little is known about their choleretic properties. In a previous paper (7), we reported the effects of an intravenous administration of the two bile acids. Bile acids exist in low levels in the systemic circulation, and for the greater amount are found in the enterohepatic circulation.

We investigated the choleretic effects and properties of ursodeoxycholic and chenodeoxycholic acids given orally and a comparison was made with dehydrocholic acid in conscious dogs. 


\section{MATERIALS AND METHODS}

All experiments were conduced on unanesthetized, male mongrel dogs (weighing 8$12 \mathrm{~kg}$ ). The animals underwent cholecystectomy and installation of a fistula (silicon fistula with small glass tube) in the common bile duct one week prior to the start of the experiments. A total amount of $1000 \mathrm{mg} /$ day of a mixture of two bile acids (cholic acid: chenodeoxycholic acid $=10: 1$ ) was administered daily in capsule form in 2 or 3 doses to complement the bile acids in the body lost from the fistula. The dogs were deprived of food $15 \mathrm{hr}$ prior to the experiment.

Bile drained from the fistula was collected in a graduated test tube and volume of bile secreted was measured at hourly intervals for $5 \mathrm{hr}$. The samples were then stored in a deepfreezer at $-20^{\circ} \mathrm{C}$ until analyzed.

Bile acid composition in the bile samples was analyzed by a modification of the techniques originally described by Klaassen (8) and Nakayama (9) using gas-liquid chromatography. The details of the methods and procedures were described previously (7).

Biliary cholesterol was determined by the method of Uda (10) which is a modification of the method of Zuckermann and Natelson (11), and bilirubin was by the method of Malloy and Evelyn (12).

Biliary phospholipid was extracted by the method of Folch et al. (13) and analyzed by the method of Fiske and Subbarow (14).

Drugs used were ursodeoxycholic acid, chenodeoxycholic acid and dehydrocholic acid (Tokyo Tanabe Co.) which were all highly purified (more than $98.5 \%$ ). All drugs were dissolved with $1 \mathrm{~N} \mathrm{NaOH}$ aqueous solution and adjusted to $\mathrm{pH} 8.0$ with $0.1 \mathrm{~N} \mathrm{HCl}$ prior to use. Each bile acid was administered orally via a stomach tube in a volume of $10 \mathrm{ml}$ following a collection of bile during the control period.

\section{RESULTS}

The normal percentage composition of bile acids in the bile of dogs with intact gallbladder was as follows: Cholic acid $80 \%$, deoxycholic acid $15 \%$, chenodeoxycholic acid $5 \%$ and unidentified below $1 \%$. On the other hand, $12 \mathrm{hr}$ after cholecystectomy deoxycholic acid markedly decreased, the percentage composition of bile acids in the fistula bile being cholic acid $87 \%$, deoxycholic acid $5 \%$ and chenodeoxycholic acid $8 \%$, respectively. The ratio of two primary bile acids, that is, cholic acid to chenodeoxycholic acid was approximately 10:1. This ratio was maintained for 2-3 weeks after cholecystectomy by a daily complement of bile acid mixture (cholic acid: chenodeoxycholic acid=10:1).

Figure 1 shows effects of ursodeoxycholic acid $(50 \mathrm{mg} / \mathrm{kg} \mathrm{p.o.)}$ on the bile flow and composition. Ursodeoxycholic acid increased the bile flow by $70 \%$ in the first $1 \mathrm{hr}$. The concentration of phospholipid in the bile increased from 11.7 to $17.3 \mathrm{mg} / \mathrm{ml}(2 \mathrm{hr}$ ), and that of cholesterol increased from 0.21 to $0.31 \mathrm{mg} / \mathrm{ml}(3 \mathrm{hr})$. The concentration of total bile acids increased from 9.42 to $16.3 \mathrm{mg} / \mathrm{ml}(2 \mathrm{hr}$ ). On the contrary, the bilirubin concentration showed an initial rise ( $1 \mathrm{hr}$ ) followed by a marked decrease.

As for bile acid composition, ursodeoxycholic acid which could not be detected before 


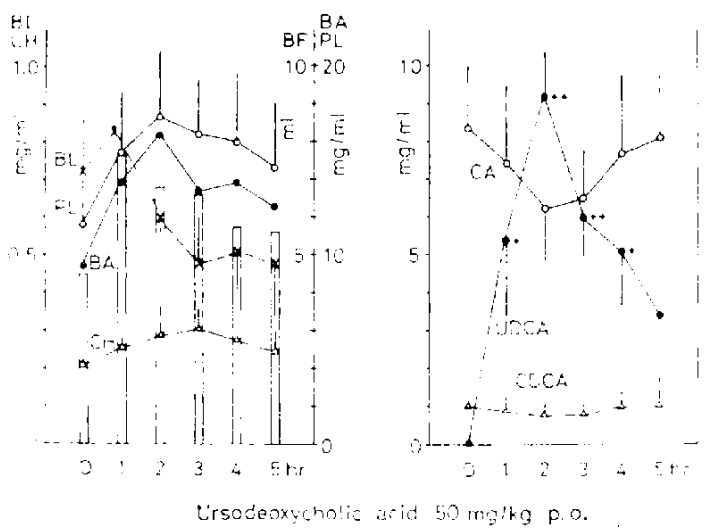

FIG. 1. Effect of ursodeoxycholic acid $(50 \mathrm{mg} / \mathrm{kg}$ p.o.) on bile flow and concentrations of bile components in conseious dogs. Left, $\mathrm{PL}=$ phospholipid $(\curvearrowright \quad$ ): $\mathrm{BL}-$ bilirubin $(\therefore \ldots \ldots)$; $\mathrm{CH}=\operatorname{cholesterol}(\triangle \quad \triangle)$; $\mathrm{BF}=$ bile flow (column):

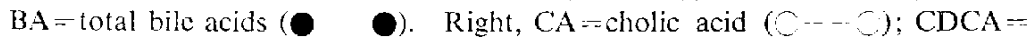
chenodeoxycholic acil (A $\mathrm{A})$; UDCA=ursodcoxycholic acid (O $)$. $p<0.05, \cdots p<0.01$; Significantly different from ' $O$ ' time (paired $t$-test). Each point represents the mean with S.E. for four experiments.

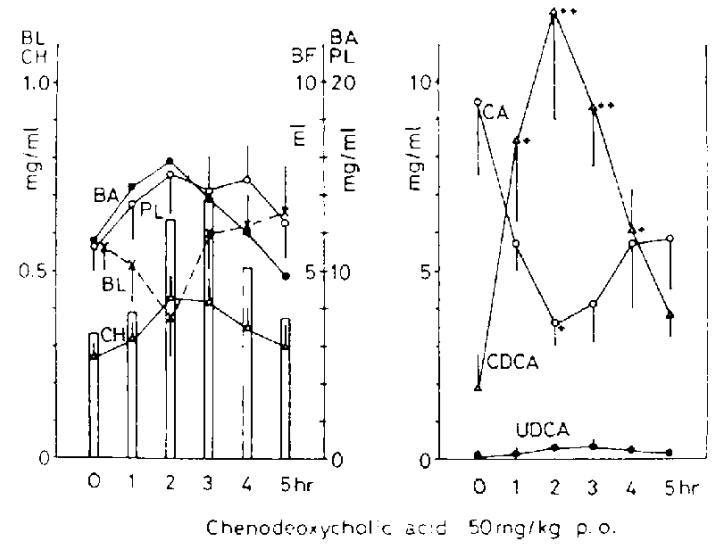

FIG. 2. Effect of chenodeoxycholic acid (50 mg/kg p.o.) on bile flow and concentrations of bilc components in conscious dogs. Notations as in Fig. 1. Each point represents the mean with S.E. for five experiments.

administration, reached a maximum concentration in the bile of $9.21 \mathrm{mg} / \mathrm{ml}$, while the two major bile acids (cholic and chenodeoxycholic acids) slightly decreased in concentrations. Total output of ursodeoxycholic acid within 5 hours was calculated to be $40 \%$ of the administered amount.

Effects of chenodeoxycholic acid (50 mg/ $/ \mathrm{kg}$ p.o.) on the bile flow and composition are shown in Fig. 2. Chenodeoxycholic acid produced a 2.1 -fold increase in bile flow $(3 \mathrm{hr}$ ). The concentration of phospholipid in the bile increased from 11.3 to $15.1 \mathrm{mg} / \mathrm{ml}$ and cholesterol concentration increased from 0.27 to $0.43 \mathrm{mg} / \mathrm{ml}(2 \mathrm{hr})$. On the other hand, the bilirubin concentration decreased for the first $2 \mathrm{hr}$. The concentration of chenodeoxycholic 


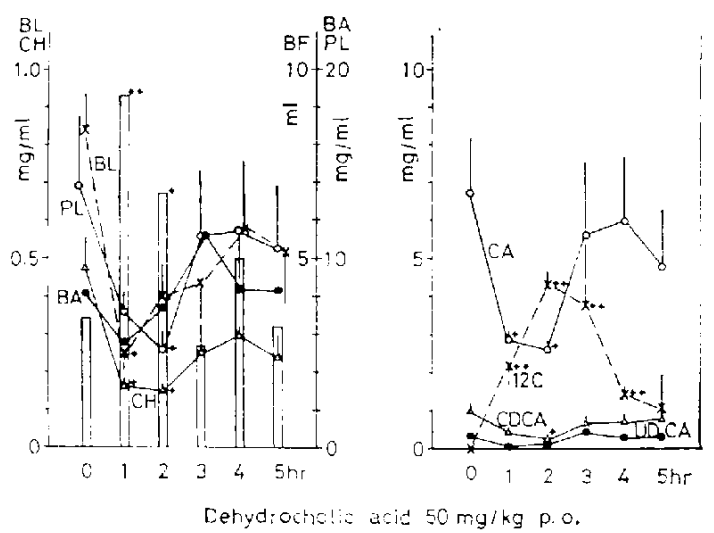

FiG. 3. Efrect of dehydrocholic acid ( $50 \mathrm{mg} / \mathrm{kg}$ p.o.) on bile flow and concentrations of bile components in conscious dogs. Notations as in Fig. 1 except $12 \mathrm{C}(3 \alpha, 7 \alpha-$ dihydroxy-12-keto-cholanoic acid $(\times \cdots \cdots \times)$ ). Each point represents the mean with S.E. for four experiments.

acid significantly increased from 1.8 to $11.4 \mathrm{mg} / \mathrm{ml}$. Ursodeoxycholic acid also slightly increased, whereas cholic acid significantly decreased from 9.5 to $3.6 \mathrm{mg} / \mathrm{ml}$. The total output of chenodeoxycholic acid was calculated to be $46^{\circ}$ of the administered amount during the first $5 \mathrm{hr}$.

Effects of dehydrocholic acid ( $50 \mathrm{mg} \mathrm{kg} \mathrm{p.o.)}$ on the bile flow and composition are shown in Fig. 3. Dehydrocholic acid produced a 2.7 -fold increase in the bile flow. In contrast, the concentration of phospholipid significantly decreased from 13.79 to $5.25 \mathrm{mg} / \mathrm{ml}$ and the cholesterol concentration significantly decreased from 0.42 to $0.13 \mathrm{mg} / \mathrm{ml}$. The bilirubin concentration significantly decreased from 0.84 to $0.25 \mathrm{mg} / \mathrm{ml}$. Total concentration of bile acids slightly decreased in the first $1 \mathrm{hr}$, but slightly increased $3 \mathrm{hr}$ after the administration. The concentrations of cholic and chenodeoxycholic acids significantly decreased. Dehydrocholic acid did not appear in the bile, but $3 \alpha, 7 \alpha$-dihydroxy-12-keto-cholanoic acid was detected and such is generally absent in the bile of dogs. Total output of $3 \alpha, 7 \alpha$-dihydroxy-12-keto-cholanoic acid within 5 hours was calculated to be $17 \%$ of the administered amount of dehydrocholic acid.

Table 1 outlines the effects of three bile acids on the total output of biliary components. Ursodeoxycholic and chenodeoxycholic acids significantly increased the total output of phospholipid. Ursodeoxycholic and chenodeoxycholic acids also increased the total output of cholesterol but there was no significant difference. Chenodeoxycholic acid increased the total output of bilirubin. On the other hand, dehydrocholic acid decreased the output of the three compounds.

Effects of three bile acids, given orally, on the total output of bile acids into the bile are shown in Table 2. Administration of ursodeoxycholic acid did not alter the output of cholic and chenodeoxycholic acids. Chenodeoxycholic acid decreased the total output of cholic acid, and dehydrocholic acid decreased the output of cholic and chenodeoxycholic acids. 


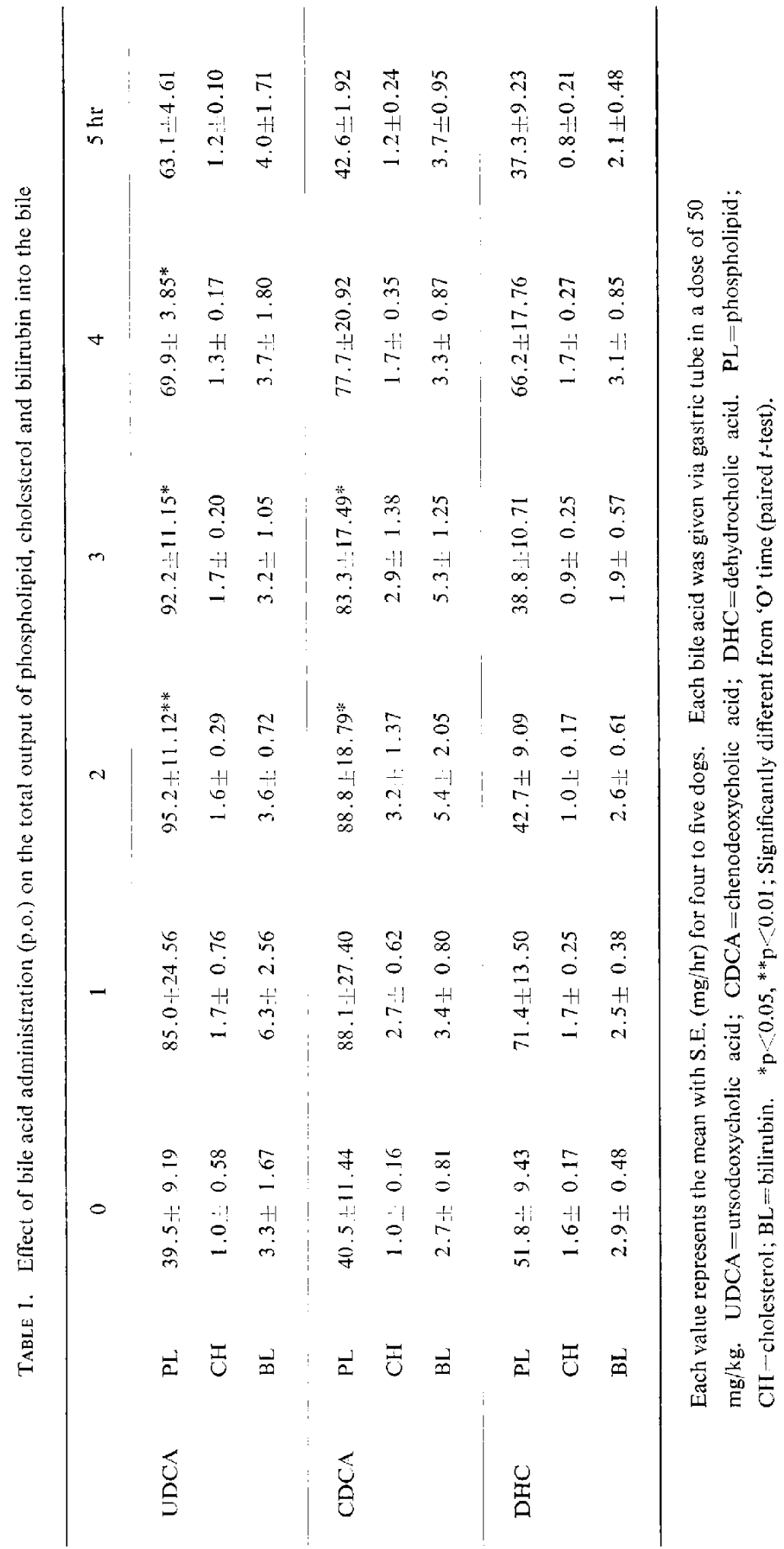


TABLE 2. Effect of bile acid administration (p.o.) on the total output of bile acids into the bile

\begin{tabular}{lccccc}
\hline & $\begin{array}{c}\text { Time } \\
\text { (hr) }\end{array}$ & CA & CDCA & UDCA & 12C \\
UDCA & 0 & $28.1 \pm 6.12$ & $4.9 \pm 2.54$ & $0.0 \pm 0.03$ & - \\
& 1 & $31.8 \pm 2.80$ & $4.6 \pm 1.43$ & $63.8 \pm 51.88$ & - \\
& 2 & $32.6 \pm 2.08$ & $4.9 \pm 1.55$ & $62.7 \pm 22.79^{*}$ & - \\
& 3 & $36.1 \pm 5.95$ & $5.6 \pm 2.03$ & $35.6 \pm 8.21^{* *}$ & - \\
& 4 & $33.7 \pm 5.75$ & $5.3 \pm 1.86$ & $21.3 \pm 3.65^{* *}$ & - \\
& 5 & $36.8 \pm 5.82$ & $5.4 \pm 1.92$ & $13.8 \pm 5.81$ & - \\
\hline CDCA & 0 & $37.8 \pm 11.50$ & $7.9 \pm 4.34$ & $1.1 \pm 0.42$ & - \\
& 1 & $36.6 \pm 10.72$ & $45.7 \pm 19.39$ & $1.3 \pm 0.51$ & - \\
& 2 & $30.3 \pm 11.15$ & $94.4 \pm 28.83^{*}$ & $3.0 \pm 2.03$ & - \\
& 3 & $23.3 \pm 4.23$ & $90.5 \pm 35.84^{*}$ & $4.2 \pm 1.83$ & - \\
& 4 & $29.1 \pm 8.72$ & $33.1 \pm 7.95^{*}$ & $1.7 \pm 0.77$ & - \\
DHC & 5 & $22.6 \pm 4.90$ & $13.7 \pm 2.15$ & $1.0 \pm 0.57$ & - \\
& 0 & $26.5 \pm 4.47$ & $5.0 \pm 1.82$ & $1.2 \pm 0.34$ & $0.0 \pm 0.00$ \\
& 1 & $28.4 \pm 2.74$ & $5.9 \pm 2.04$ & $1.2 \pm 0.44$ & $17.2 \pm 5.44^{*}$ \\
& 2 & $19.6 \pm 2.56$ & $3.7 \pm 2.07$ & $0.8 \pm 0.32$ & $30.2 \pm 2.84^{* *}$ \\
& 3 & $16.1 \pm 4.36$ & $2.9 \pm 1.29$ & $0.9 \pm 0.48$ & $16.2 \pm 6.75^{*}$ \\
& 4 & $26.5 \pm 5.35$ & $4.6 \pm 1.39$ & $1.2 \pm 0.66$ & $13.1 \pm 5.96^{*}$ \\
& 5 & $15.2 \pm 4.02$ & $3.1 \pm 1.20$ & $0.9 \pm 0.40$ & $5.9 \pm 2.83^{*}$ \\
\hline
\end{tabular}

Each value represents the mean with S.E. (mg/hr) for four to five dogs. Each bile acid was given via gastric tube in a dose of $50 \mathrm{mg} / \mathrm{kg}$. UDCA=ursodeoxycholic acid; $\mathrm{CDCA}=$ chenodeoxycholic acid; $\mathrm{DHC}=$ dehydrocholic acid; $\mathrm{CA}=$ cholic acid. $12 \mathrm{C}=3 \alpha, 7 \alpha$-dihydroxy-12-keto-cholanoic acid. - : not detected. ${ }^{*} \mathrm{p}<0.05$, ** $\mathrm{p}<0.01$; Significantly different from ' $\mathrm{O}$ ' time (paired $t$-test).

\section{DISCUSSION}

Ursodeoxycholic acid increased the bile flow and the concentrations of phospholipid, cholesterol and ursodeoxycholic acid in the bile, suggesting that ursodeoxycholic acid has a choleretic effect associated with secretion of biliary components such as phospholipid and cholesterol, and the choleretic activity which is induced by ursodeoxycholic acid itself is secreted in high concentrations from the hepatic parenchymal cells into the bile canaliculi. Chenodeoxycholic acid was found to have a similar mode of action to that of ursodeoxycholic acid.

On the other hand, dehydrocholic acid, unlike ursodeoxycholic and chenodeoxycholic acids, markedly decreased the concentrations and total output of phospholipid and cholesterol despite a significant increase in the volume of bile flow. Thus it was confirmed that dehydrocholic acid is a choleretic which induces secretion of water and electrolytes from the bile canaliculi, and is not accompanied by secretions of phospholipid and cholesterol from hepatic cells.

The mechanisms of bile formation are generally classified into the bile acid-dependent fraction and bile acid-independent one. The water in the bile is, reportedly, derived from both fractions $(3,15)$. Phospholipid and cholesterol excretions into the bile are bile acid- 
dependent (2). On the other hand, electrolyte excretions into the bile are considered to be exclusively bile acid-independent $(3,4,5)$. Bilirubin excretion is also reportedly bile acid-independent $(16,17)$.

The present findings indicate that both ursodeoxycholic and chenodeoxycholic acids act on the hepatic cells and play a role in the bile acid-dependent mechanism of the bile formation. On the other hand, dehydrocholic acid is probably metabolized in the hepatic cells into $3 \alpha, 7 \alpha$-dihydroxy-12-keto-cholanoic acid and the metabolite produces hydrocholeresis. Dehydrocholic acid is metabolized mainly into the dihydroxy-monoketo bile acid in humans (18).

\section{REFERENCES}

1) Preisig, R., Cooper, H.L. ANd Wheeler, H.O.: The relationship between taurocholate secretion rate and bile production in the unanesthetized dog during cholinergic blockade and during secretin administration. J. clin. Invest. 41, 1152-1162 (1962)

2) WheEler, H.O. AND King, K.K.: Biliary excretion of lecithin and cholesterol in the dog. J. clin. Invest. 51, 1337-1350 (1972)

3) Erlinger, S. ANd Dhumeaux, D.: Mechanisms and control of secretion of bile water and electrolytes. Gastroenterology 66, 281-304 (1974)

4) Whieler, H.O., Ross, E.D. ANd Bradley, S.E.: Canalicular bile production in dogs. Am. J. Physiol. 214, 866-874 (1968)

5) Erlinger, S., Dhumeaux, D., Berthelot, P. And Dumont, M.: Effect of inhibitors of sodium transport on bile formation in the rabbit. Am. J. Physiol. 219, 416-422 (1970)

6) KlaAsSEN, C.D.: Comparison of the choleretic properties of bile acids. Europ. J. Pharmacol. 23, 270-275 (1973)

7) Yanaura, S. AND ISHIKAWA, S.: Effects of some bile acids on biliary flow and composition in dogs. Folia pharmacal. japon 72, 689-700 (1976) (Abs. in English)

8) KLAASSEN, C.D.: Gas-liquid-chromatographic determination of bile acids in bile. Clin. Chim. Acta 35, 225-229 (1971)

9) Nakayama, F.: Quantitative microanalysis of bile. J, Lab. clin. Med. 69, 594-609 (1967)

10) UDA, T.: Cholesterol content of the cystic bile, hepatic bile and serum in patients with hepatobiliary discasc. Geka no Ryoiki 8, 163-181 (1960) (in Japanese)

11) ZUCKERMANN, J.L. AND NATflson, S. : A convenient and rapid procedure for total cholesterol estimation with an acid-chloroform extraction. J. Lab. clin. Med. 33, 1322-1325 (1948)

12) Malloy, H.T. AND Evelyn, K.A.: The determination of bilirubin with the photoelectric colorimeter. J. biol. Chem. 119, 481-490 (1937)

13) Folch, J., Lees, M. and Sloane Stanley, G.H.: A simple method for the isolation and purification of total lipids from animal tissues. J. biol. Chem. 226, 497-509 (1957)

14) Fiske, C.H. A.ND Subbarow, Y.: The colorimetric determination of phosphorus. J. biol. Chem. 66, 375-400 (1925)

15) WHEELER, H.O.: Secretion of bile acids by the liver and their role in the formation of hepatic bile, Arch. intern. Med. 130, 533-540 (1972)

16) Alpert, S., Mosher, M., Silanske, A. and Arias, I.M.: Multiplicity of hepatic excretory mechanisms for organic anions. J. gen. Physiol. 53, 238-247 (1969)

17) Shani, M., Seligsohn, U. A.jd Ben-Ezzer, J.: Effect of phenobarbital on liver functions in patients with Dubin-Johnson syndrome. Gastroenterology 67, 303-308 (1974)

18) Soloway, R.D., Hofmani, A.F., Thomas, P.J., Schoenfield, L.J. and Klein, P.D.: Triketocholanoic (Dehydrocholic) acid. Hepatic metabolism and effect on bile flow and biliary lipid secretion in man. I. clin. Invest. 52, 715-724 (1973) 